\title{
EL ROL DE LOS ASPECTOS EMOCIONALES EN LA GESTIÓN DE LA CULTURA INNOVADORA*
}

\author{
MARIA ISABEL CAMIO** \\ UNIVERSIDAD NACIONAL DEL CENTRO DE LA PROVINCIA DE BUENOS AIRES - UNICEN (ARGENTINA)
}

Recibido/ Received/ Recebido: 06/12/2013 - Aceptado/ Accepted / Aprovado: 29/05/2014

\begin{abstract}
Resumen
Este trabajo se enmarca en una investigación más amplia (Camio, 2012), enfocándose en el estudio de la cultura para la generación de innovación, incorporando la perspectiva emocional en el análisis. Se trata de un estudio cualitativo, un estudio de caso en profundidad de una empresa de software del Polo de Software de la Universidad Nacional del Centro de la Provincia de Buenos Aires (UNI$\mathrm{CEN}$ ), Argentina. En este documento se exponen las características generales del caso en estudio, se aplican los lineamientos para la identificación de paradigmas culturales a partir de las presunciones constituyentes y su interrelación, y se analizan los resultados de la aplicación de un índice del nivel de receptividad de la cultura de los aspectos emocionales relacionados con la creatividad y la innovación (INCEIC). Esta propuesta profundiza y enriquece el estudio de la cultura organizacional, porque la metodología de operacionalización propuesta contribuye al diagnóstico de la capacidad creativa de los individuos y del potencial para generar innovación sostenida.
\end{abstract}

Palabras clave: Innovación, Cultura, Emociones, Índice.

\section{ROLE OF EMOTIONS IN INNOVATIVE CULTURE MANAGEMENT}

\begin{abstract}
This paper is part of a wider research (Camio, 2012), which focuses on the study of culture for innovation generation and incorporates an emotional perspective in the analysis. This was a deep qualitative case study of a software's company from Polo software of the Universidad Nacional del Centro de la Provincia de Buenos Aires (UNICEN) in Argentina. Its general characteristics are presented, and from fundamental assumptions and their interrelationships the guidelines for cultural paradigms identification are applied. The results from the implementation of the index of culture responsiveness level of emotional aspects related to creativity and innovation (INCEIC) are analyzed. This proposal deepens and enhances organizational culture study because the proposed operationalization methodology contributes to a proper diagnosis of individual's creative capacity and potential to generate sustained innovation.
\end{abstract}

Keywords: Innovation, Culture, Emotions, Index.

\footnotetext{
Artículo derivado del proyecto de investigación titulado "Innovación y Modalidades de Gestión", adelantado por el Centro de Estudios en Administración de la Facultad de Ciencias Económicas - Universidad Nacional del Centro de la Provincia de Buenos Aires - UNICEN, entre los años 2012 - 2014.

** Profesora de grado y postgrado del Área Teoría de la Organización de la Facultad de Ciencias Económicas de la Universidad Nacional del Centro de la Provincia de Buenos Aires - UNICEN. Doctora en Administración de Universidad Nacional de Rosario. MBA, Magíster en Comercialización, Contador Público y Licenciada en Administración de la UNICEN. Co-directora del proyecto"Innovación y Modalidades de Gestión" del Centro de Estudios en Administración del (CEA) y Directora del Programa GUIA - Gestión de la Innovación y el Aprendizaje de la UNICEN. Correo electrónico: camio@econ.unicen.edu.ar. Tel: (54) 02494385550 int. 4325. Dirección postal: Arroyo Seco s/n- Campus Universitario- Universidad Nacional del Centro de la Provincia de Buenos Aires- (7000) Tandil Argentina.
} 


\title{
O PAPEL DOS ASPECTOS EMOCIONAIS NA GESTÃO DA CULTURA INNOVADORA
}

\begin{abstract}
Resumo
Este trabalho está contextualizado numa pesquisa mais ampla (Camio, 2012) que se enfoca no estudo da cultura para a geração de inovação e incorpora a perspectiva emocional na análise. A pesquisa foi um estudo de caso de ordem qualitativa em profundidade de uma empresa de software do Polo de Software da Universidade Nacional do Centro da Província de Buenos Aires (UNICEN), Argentina. Apresentam-se as características gerais do caso em estudo, aplicam-se as linhas para a identificação de paradigmas culturais a partir das presunções constituintes e sua inter-relação; e se analisam os resultados da aplicação de um índice do nível receptividade da cultura dos aspectos emocionais relacionados com a criatividade $e$ a inovação (INCEIC). Esta proposta aprofunda $e$ enriquece o estudo da cultura organizacional porque a metodologia de operacionalização proposta contribui com um adequado diagnóstico da capacidade criativa dos indivíduos e do potencial para geração de inovação sustentada.
\end{abstract}

Palavras chave: Inovação, Cultura, Emoções, Índice.

Camio, M. (2014) El rol de los aspectos emocionales en la gestión de la cultura innovadora. En: Revista de la Facultad de Ciencias Económicas de la Universidad Militar Nueva Granada. rev.fac. cienc.econ, XXII (2).

JEL: O31, O32, O39.

\section{Introducción}

A partir de considerar los hallazgos de diferentes estudios que correlacionan la gestión de la innovación con el desempeño de las organizaciones/empresas y entendiendo que la cultura opera como condicionante al momento de aprovechar los conocimientos y capacidades para la gestión de la innovación surgen interrogantes que direccionan los esfuerzos de investigación.

En primer término, resulta necesario indagar acerca de la relación entre la creatividad y la innovación en el ámbito de la organización, y en qué nivel de análisis se anclan cada una de las mencionadas variables. Sin desconocer la interrelación entre el proceso creativo y la gestión de la innovación resulta una definición propia apoyada en otros estudios anteriores, la consideración de la creatividad como una variable a nivel individual y la innovación como un proceso a ser analizado a nivel organizacional.

Por otra parte se enfatiza en las preguntas acerca de qué enfoques de la creatividad/innovación resultan adecuados para contextos de complejidad, donde el dinamismo imperante requiere respuestas adecuadas a estos contextos y con ajustes temporales estrechos.

Si se considera a la cultura como un condicionante (positivo o negativo) para la gestión de la innovación, surge un elemento central a tener en cuenta el referido a la necesidad de contar con herramientas de diagnóstico adecuadas en términos de profundidad e integralidad de su abordaje. Surgen así preguntas que se entienden requieren una respuesta: ¿Qué dimensiones de análisis resultan relevantes? ¿Qué metodologías resultan apropiadas en términos de la complejidad, particularidad, profundidad del fenómeno de la cultura? ¿Cómo se articulan las respuestas requeridas en una cultura que impulse la innovación?

A partir de señalar los cuestionamientos que direccionan el presente trabajo, se exponen en el marco teórico las definiciones del fenómeno de innovación organizacional, se recepta una postura paradigmática de la cultura como metáfora fundamental, se 
refiere a estudios que vinculan los procesos creativos y de innovación en el ámbito organizacional. Se señalan investigaciones que destacan la necesidad de considerar aspectos emocionales en la gestión, haciendo hincapié en los estudios sobre creatividad y generación de innovación empresarial.

En el apartado de metodología se exponen los elementos distintivos de la misma que señalan sus características particulares, las variables a considerar en el análisis, sus categorías de respuesta y la identificación de las mismas como barreras o impulsores de la creatividad/innovación. Se aplica la metodología propuesta en un estudio de caso en profundidad, realizando un análisis del nivel de articulación del paradigma cultural y de asociación con la innovación. Se exponen los resultados a nivel de variables componentes y se calcula el INCEIC en la empresa del caso. En el acápite final, se señalan los aspectos conclusivos del presente trabajo y se plantean lineamientos para futuros estudios en la temática.

\section{Marco conceptual}

En la construcción del marco que articule los conceptos y variables incluidas en este estudio, se destaca la relación de la innovación con el desarrollo económico, lo que justifica el estudio de este fenómeno a nivel organizacional; se expone el concepto de innovación a aplicar y se puntualiza la relación de las empresas con el entorno (concepto de innovación abierta) lo que requiere de una cultura con características de apertura al entorno. A continuación se analiza la relación innovación-creatividad, a fin de dar cuenta de tales procesos en términos de individualización de los mismos y de su articulación.

En el segundo acápite se introduce la variable cultura en términos conceptuales y se señala el abordaje elegido para el desarrollo de la investigación. Como justificación de la inclusión de los aspectos emocionales en la metodología a proponer se citan trabajos que señalan la importancia de investigar el impacto de las emociones en la gestión y particularmente en el estudio de la cultura organizacional.

Se receptan los autores referentes en relación a los aspectos emocionales en las organizaciones, y se introduce un modelo para el desarrollo de la inteligencia emocional aplicado en algunas de las subvariables del índice a proponer.

En último término se refieren varios estudios contemporáneos que señalan hallazgos que confirman la relación entre el desarrollo de capacidades emocionales y la innovación organizacional.

\subsection{Creatividad e innovación en el ámbito organizacional}

La innovación se entiende como un proceso que facilita la competitividad empresarial y contribuye al desarrollo económico de las sociedades (Lundvall \& Maskell, 2000; Yoguel \& Boscherini, 1996).

Entender a la innovación como capacidad dinámica, refiere a "la capacidad de la organización de crear, extender o modificar su base de recursos intencionalmente" (Helfat et al., 2007, 4) por la adición de nuevo conocimiento en los nuevos productos, servicios, procesos, tecnologías o métodos de gestión.

Una visión amplia de la innovación considera a esta como una operación empresarial que impregna a toda la empresa y no se restringe a un área específica $e$ involucra la interacción con el entorno. En particular, se entiende a la innovación empresarial como un proceso de cambio, tanto incremental como sustancial, en productos, procesos, organización y/o mercadotecnia (Cotec, 2006).

Haciendo foco en la relación organización/contexto, surge una perspectiva amplia de los tipos de intermediación requeridos, como son la construcción de redes, el sostenimiento del aprendizaje social y la gestión de la dinámica de poder y los conflictos (Leeuwis \& Aarts, 2011).

Chesbrough (2003) introdujo la idea de innovación abierta en el año 2003, como un modo de aumentar el número de ideas utilizando la capacidad de innovación que existe fuera de la propia organización. Durante la era moderna de la innovación abierta, las personas en diferentes roles, por ejemplo como visionarios, innovadores, líderes, empleados, clientes e incluso el ciudadano común han sido con- 
siderados, como creativos si tienen el potencial para desarrollar ideas innovadoras (Hirvikoski \& Diz, 2009); por otra parte, también se destaca el rol relevante de las redes de contacto en las distintas etapas del proceso emprendedor (Kantis, 2004, 48).

Hirvikoski \& Diz (2009) llevando el tema al ámbito laboral, agregan que, al contratar personas, los empleadores buscan a aquellos que poseen potencial de innovación, y trabajan sólo de forma activa en el fomento de un entorno en que el pensamiento creativo se nutre del potencial de innovación.

Csikszentmihalyi $(2011,21)$ sugiere que la creatividad es el resultado de un sistema compuesto por tres elementos: una cultura que contiene reglas simbólicas, una persona que aporta novedad al campo simbólico y un ámbito de expertos que reconocen y validan la innovación. En su obra, Hirvikoski \& Diz (2009) afirman que se puede ser creativo sin ser innovador, pero no se puede ser innovador sin ser creativo.

Resulta un llamado de atención lo expuesto por Conger (1989), cuando afirma que "Es un error pensar que los líderes son los genios creativos detrás de sus ideas. Muchas veces su creatividad deviene de su apertura a la creatividad de los demás y la comprensión de las implicaciones prácticas”.

Por otra parte, la creatividad empresarial se relaciona a una concepción que la asocia con la innovación. En este sentido Morcillo (1997, 106) señala que la "creatividad permite usar de manera original el conocimiento disponible con el propósito de obtener unas innovaciones que mejoraran el posicionamiento competitivo de las compañías". En este sentido, para estimular la creatividad es primordial contar con una cultura empresarial, con un entorno de trabajo que facilite la innovación ya que "quien disfruta de lo que hace, quien ama su trabajo y goza con el aprendizaje tiende a la creatividad y no a la rutina" (Morcillo, 1997, 135).

La creatividad se puede entender como una función de las características individuales y los factores contextuales/sociales (Amabile et al., 1996; Gough, 1979; Oldham \& Cummings, 1996; Scott \& Bruce, 1994; Woodman et al., 1993 citados por Lim
\& Choi, 2009). Para comprender mejor el proceso creativo, algunos investigadores han propuesto que los factores individuales y sociales pueden promover la creatividad a través de procesos psicológicos tales como libertad psicológica y sensibilidad a las oportunidades para mejorar (Hennessey \& Amabile, 1988 citados por Lim \& Choi, 2009).

En el análisis de la innovación es necesario identificar las variables organizacionales que operan como condicionantes. Se destaca que en el proceso de innovación confluyen distintos conocimientos y capacidades que están presentes en las diferentes áreas de la empresa, cuyo aprovechamiento depende de la cultura organizacional de la firma (Yoguel \& Boscherini, 1996, 5).

\subsection{La cultura y sus abordajes}

El abordaje del fenómeno cultural ha sido realizado desde distintas disciplinas, como son la antropología, la sociología, la psicología social, entre otras. La administración ha avanzado con desarrollos propios y se ha nutrido, en algunos casos, de los enfoques propuestos por las otras disciplinas.

Se comparte el enfoque que considera que la organización es cultura (Dávila \& Martínez, 1999). Esta posición resulta consistente con una metodología cualitativa para el diagnóstico cultural. El análisis se centra en las particularidades de las empresas a estudiar, aunque se tiene en cuenta el sector de negocios y el país a que pertenecen las mismas.

El enfoque analítico de Schein (1988, 30) distingue tres niveles para el análisis de la cultura, que comienza con el más superficial, al que denominó "artefactos y creaciones visibles pero difíciles de descifrar", un segundo nivel al que denominó "valores", los que, una vez haber transitado el proceso de confrontación pasan a formar parte del nivel más profundo de análisis, el de las "presunciones básicas" invisibles $y$ preconscientes, dadas por sentadas por los individuos que forman parte de la organización. Este enfoque fue tomado como base para el desarrollo del trabajo propuesto, por lo que se retomarán algunos elementos base de su construcción teórica en apartados subsiguientes. 
El aspecto más difícil del análisis de las presunciones atañe (Schein, 1988, 117) a la escala en que llegan a constituirse como "paradigmas" o esquemas coherentes. No todas las presunciones son mutuamente compatibles o congruentes.

Las presunciones se dan en distintos ámbitos, algunos de los cuales son más superficiales y periféricos que otros. Al momento de definir teóricamente los paradigmas, se toman de acuerdo a diferentes categorías, derivándose más directamente unas de otras en función de su nivel de jerarquía.

En este punto del análisis se recepta lo propuesto por Rodríguez (2006, 270), entendiéndose que las premisas del decidir que conforman la cultura organizacional no son necesariamente producto de la experiencia, ni mucho menos de la experiencia exitosa. Tampoco son coherentes entre sí -se entiende para este trabajo que no necesariamente son coherentes entre sí-. A pesar de esto, el autor plantea que la cultura opera como un reservorio de experiencias y sabiduría, lo que hace difícil su cambio.

En el estudio de las capacidades para generación de la innovación y haciendo foco en la cultura que impulsa la generación de la creatividad y potencia la innovación a nivel de la organización, el enfoque de Schein (1988) realiza un importante aporte a partir de la profundidad de su modelo de análisis, y su desarrollo metodológico, aunque se entiende necesario considerar el aporte de otros enfoques más amplios desde su perspectiva de análisis, como es el metafísico de Owen (Lessem, 1992, 185), debido a la pretensión de interpretar en el análisis organizacional, al individuo desde su integralidad.

\subsection{Los aspectos emocionales en la gestión}

El estudio de los aspectos emocionales en el trabajo comienza en 1930 y hasta 1990 no se presentan avances importantes en esta área de estudio (Brief $\&$ Weiss, 2002). Algunos estudios se concentran en el análisis de los modelos de medición de la Inteligencia Emocional (Cartwright \& Pappas, 2007) y, en el análisis de cómo la regulación de las emociones impacta en la presión laboral (Côté, 2005).
En 1998 tiene lugar la First Conference on Emotions and Organizational Life, donde se publica, entre otros avances, un clúster de discusión (Fisher \& Ashkanasy, 2000, 5) de temas vinculados a la investigación de las emociones en el trabajo. En la lista de tópicos (Zerbe, 1997, citado por Fisher \& Ashkanasy, 2000) para futuras investigaciones se agrupan en ítems como determinantes de las emociones, naturaleza y descripción de las emociones. Por otra parte se señalan distintos subtemas agrupados por niveles de análisis: el individual, el grupal y el organizacional. En el nivel organizacional se señala como tópico de interés para investigaciones futuras el rol de las emociones en la cultura y el clima organizacional.

En el estudio de los aspectos emocionales surgen investigaciones disruptivas en términos de su consideración en la gestión. A partir de hallazgos derivados de investigaciones en el campo de la neurobiología (Damasio, 2001, 182), las emociones no se entienden como una limitante sino como un prerrequisito para la acción de decidir satisfactoriamente. Lo anterior guarda relación con los estudios de Kahneman (2011) desde el campo de estudio de la teoría de la decisión.

Kahneman $(2011,13)$ con el objetivo de introducir un idioma para pensar y actuar acerca de la mente, utiliza la metáfora de dos agentes ficticios llamados Sistema I y Sistema II, los cuales respectivamente producen pensamiento rápido y lento.

Damasio (2000, 58) sostiene que emociones y sentimiento de emociones, respectivamente, son principio y fin de una misma progresión. Distingue entre emociones primarias, secundarias y sentimientos. Propone el término emoción para connotar la combinación de un proceso que produce un estado corporal y emocional, con cambios mentales adicionales. Se deja el término "sentimientos" para la experimentación de los cambios.

Elster $(2001,39)$ propone que las emociones pueden provocarse por gran diversidad de creencias, que pueden tener relación con los otros o con uno mismo, con el pasado, el presente y el futuro; y estas creencias puede que las consideremos como ciertas, probables o simplemente posibles. En este sentido, 
hace hincapié en tres características de las emociones: i) son el soporte principal de las normas sociales; ii) no todas las culturas reconocen o conceptualizan las mismas emociones; iii) cuando una emoción está integrada en el repertorio conceptual de una cultura, puede convertirse también en el objeto de normas sociales, lo que conduce a que la emoción se produzca de manera más o menos frecuente (Elster, 2001, 98).

Ya en el campo de la gestión, Cooper \& Sawaf (1998) proponen su modelo para el desarrollo de la Inteligencia Emocional con cuatro pilares. Su propuesta aparta la inteligencia emocional del campo del análisis psicológico y la coloca en el terreno del conocimiento directo, el estudio y la aplicación a las organizaciones. Los cuatro pilares propuestos fueron: i) el conocimiento emocional, ii) la aptitud emocional, iii) la profundidad emocional, y iv) la alquimia emocional.

En el último de los pilares, la alquimia emocional (Cooper \& Sawaf, 221-287) se propone la búsqueda de la confluencia, que es la reunión de intuiciones y talentos dispares, propósitos y competencias, personas y posibilidades, en un todo unificado. El flujo intuitivo posibilita encontrar el punto clave donde actuar. Se señala al sentido del humor como la conducta más significativa de la inteligencia humana, la que aumenta la energía y fomenta el flujo intuitivo, mejora los procesos de inteligencia, como el juicio, la solución de problemas y la toma de decisiones en circunstancias difíciles. Es una gran ayuda para la transformación creativa. La alegría es irreverencia y hace al cambio menos atemorizante. Recientes estudios enfatizan el rol del sentido del humor para la mejora de la performance creativa en el lugar de trabajo (Lang \& Lee, 2010).

Por otra parte, es necesario destacar que los estudios sobre creatividad de los individuos, han avanzado en la consideración de aspectos emocionales para la evaluación de la capacidad creativa. El ECI es un cuestionario que se puede utilizar para medir diferencias individuales de creatividad emocional y se centra en dos de las etapas del proceso creativo propuesto por Wallas (citado por Averill, 1999): preparación y verificación. En las variables a tener en cuenta para identificar el nivel de capacidad creativa de los individuos se propone entre los hábitos de trabajo el grado de uso de la multidimensionalidad en la toma de decisiones (Camio et.al, 2013).

En el análisis de las relaciones entre los aspectos emocionales y la generación de la innovación empresarial, se toman en consideración diferentes estudios. Ashforth \& Humphrey (1995) señalan que ciertas tareas organizacionales -por ej. procesos creativos y aprendizaje- pueden beneficiarse por un ambiente que permita la expresión y discusión de las emociones.

Por otra parte, Choi, Sung, Lee \& Cho (2010) proponen un modelo de investigación de la efectividad en la implementación de la innovación que considera tanto los procesos cognitivos como emocionales. Los autores avanzan en su propuesta a partir de la articulación de otros estudios (Weiner, 1986; Huy, 2002; Lewis, Sullivan \& Michalson, 1984; Weis \& Cropanzano, 1996; Davis, 1989; Liu \& Perrewe, 2005 citados por Choi et al., 2010).

A partir de la investigación realizada, y con las limitaciones expuestas en ese trabajo (Choi, et al., 2010), se evidencia que los empleados que comparten el mismo ambiente de trabajo tienden a compartir las evaluaciones tanto cognitivas como emocionales acerca de la innovación. Se puntualiza la necesidad de estudios futuros del impacto de los aspectos emocionales en la etapa de implementación de las innovaciones.

Otros estudios vinculan las capacidades de aprendizaje, las capacidades emocionales y la innovación de productos. Akgün, Keskin, Byrne \& Aren (2007) siguiendo a Jerez, Céspedes \& Valle (2005), entienden la capacidad de aprendizaje como un concepto complejo y multidimensional compuesto por: i) el compromiso del Management, ii) la perspectiva sistémica, iii) la apertura y la experimentación, y iv) la transferencia e integración de conocimientos.

Por otra parte, Akgün et al. (2007) operacionalizan la variable capacidad emocional a nivel organizacional a partir de la utilización de cuatro de las dinámicas propuestas por Huy para definir "dinámica emocional", a saber, i) la libertad de auténtica expresión de 
las emociones, ii) la cualidad de una organización de hacer esfuerzos por identificar la variedad de emociones, aceptarlas, internalizarlas, y profundizar su comprensión, iii) la dinámica de la reconciliación que permite unir los valores aparentemente opuestos de los individuos y generar puentes entre sus emociones, iv) la dinámica de la identificación, se refiere al fuerte apego a la identidad y las características salientes de la organización, por la cual los participantes están juntos por beneficios mutuos y vínculos emocionales.

A partir de los hallazgos obtenidos (Akgün et al., 2007) se puede afirmar que la capacidad emocional de las empresas influencia sus capacidades de aprendizaje e indirectamente la capacidad de innovar en productos y la performance organizacional.

Tomando como base los trabajos de Nayak \& Katteringham (1986), que argumentan que el espíritu y la emoción dentro de los individuos son el combustible para la creación de innovaciones radicales, Sandberg (2007) señala que se puede relacionar el éxito en el lanzamiento de este tipo de innovaciones con el nivel de entusiasmo generado en la empresa -pasión por participar en una actividad en particular, por el disfrute o por considerarlo importante-.

En el estudio realizado surge que en las etapas de generación de ideas parece no estar presente ningún inhibidor del entusiasmo, porque es la etapa en donde se requiere creatividad e inspiración. Brechas de compromiso, confianza, involucramiento y resultados surgen en la etapa de desarrollo. En este estudio surge además, como variable de contingencia el tamaño, debido a que las entrevistas indicaron que el entusiasmo se propaga naturalmente en pequeñas organizaciones resultado de la comunicación interfuncional más frecuente e informal.

En otro estudio realizado por Baron \& Tang (2011), se propone un modelo que señala la relación entre las emociones positivas de los empresarios y los aspectos importantes de la creación de nuevos emprendimientos. En primer lugar, porque los empresarios operan en ambientes de incertidumbre y de alta tasa de cambio. En segundo lugar, se ha verificado que las emociones influyen fuertemente en el lan- zamiento y operación de las nuevas empresas -en la toma de decisiones y el juicio, formando relaciones positivas de trabajo, la persuasión y la obtención de un alto nivel de entusiasmo, en otros- (Diener \& Seligman, 2002; Forgas, 2000 citados por Baron \& Tang, 2011). Los resultados de la citada investigación proporcionan evidencia empírica de una relación entre la creatividad y la innovación, y agregan a nuestra comprensión de los procesos complejos a través de los cuales las variables a nivel individual como la creatividad y las emociones positivas en último instancia, influyen en los resultados a nivel organizacional como la innovación.

\section{Metodología}

En este trabajo se considera que la organización es una cultura y que para su comprensión se requiere descubrir los aspectos subyacentes, que han sobrevivido a los procesos de confrontación tanto físicos como sociales. Se propone el análisis del fenómeno cultural desde una perspectiva analítica no racional pura, sino desde una perspectiva integral que contemple aspectos emocionales, con el objetivo de diagnosticar la capacidad de la organización para generar procesos sostenidos de innovación.

Al momento del estudio de la cultura organizacional, se destacan entre los factores de contingencia que afectan a la variable cultura, el tamaño de la organización, las fuerzas del entorno -en especial de su sector de negocios-, y las características de los individuos participantes, entre las que se destacan la posición de poder (Fitness, 2000).

Se propone identificar el nivel de coherencia o la falta de esta en las respuestas obtenibles en las dimensiones de análisis a partir de su articulación o no en paradigmas culturales. Para su estudio, la metodología a emplear tiene características cualitativas, a través de estudios en profundidad donde las categorías para su identificación resultan estandarizables a los fines comparativos entre diferentes empresas pero que adquieren significado particular en cada caso específico.

El análisis conjunto de los aspectos emocionales y racionales en el diagnóstico de la cultura organiza- 
cional resulta justificado a partir de entender que las emociones resultan un prerrequisito para la acción de decidir satisfactoriamente, que existen mecanismos que pueden producir variación cultural de las emociones y que éstas pueden modificar la actividad cognitiva.

En este estudio, la unidad de análisis (UA) es la empresa, ya que la cultura y la innovación resultan variables de este nivel. Se releva una muestra de personas de la misma, con fines netamente instrumentales o no sustanciales. El estudio de casos permite analizar el fenómeno objeto de estudio en su contexto real, utilizando múltiples fuentes de evidencia, cuantitativas y cualitativas simultáneamente. Este enfoque se entiende pertinente para avanzar en el conocimiento de determinados fenómenos complejos, como los referidos a los objetivos de este trabajo, los que se exponen a continuación:

- Definir los lineamientos para la identificación de la existencia o no de un paradigma cultural, a partir de las presunciones constituyentes y su interrelación.

- Construir un índice del nivel de receptividad de la cultura de los aspectos emocionales en relación con la creatividad y la innovación.

- Detectar la relación entre el paradigma cultural y el nivel de receptividad de la cultura de los aspectos emocionales en relación con la creatividad y la innovación en el caso en estudio.

Para dar cumplimiento al primer objetivo, es necesario considerar que en la variable cultura, cada una de las dimensiones operan como variables genuinas que ya tienen un valor de respuesta determinado, por tanto el análisis resulta centrado en la unidad de análisis, en la búsqueda de tipologías culturales en las empresas en estudio, específicamente en la búsqueda de existencia o no de paradigmas articulados y sus características propias.

Se exponen en la Tabla 1 las dimensiones (sub-variables) e indicadores, junto a las respuestas posibles y su asociación con la innovación, a fin de identificar su efecto como barrera o impulsor. Se propone el cálculo del grado de articulación del paradigma (GAP), a partir de la consideración del nivel de alineación o no de estas respuestas, es decir su nivel de coherencia. Por otra parte, se considera también su nivel de asociación con la innovación (NAI), para lo que se tiene en cuenta la ubicación de la respuesta a los indicadores en la columna respectiva y su consideración como barrera o impulsor de la innovación.

En respuesta al segundo objetivo es necesario considerar que el nivel de receptividad de la cultura de los aspectos emocionales para la generación de creatividad e innovación se encuentra en una etapa que podría llamarse exploratoria, lo que de alguna manera obliga a realizar operaciones complejas para poder proponer una clasificación que sintetice todas las dimensiones componentes. El análisis se centra en una primera instancia, en el valor (en $\mathrm{R}$ ), requiriéndose buscar la manera de sintetizar dimensiones o sub-variables, resultando necesario por tanto, realizar una "clasificación" ${ }^{-}$-como la ideación de un sistema de ordenamiento de objetos-.

El Nivel de receptividad de la cultura de los aspectos emocionales para la creatividad $e$ innovación no pudo ser relevado directamente a través de una única variable, sino que debió construirse a través de varias sub-variables, que actúan como aproximaciones para determinar su nivel (Baranger, 1995). En la segunda parte de la Tabla 1 se exponen las variables, sub-variables e indicadores propuestos.

Se realizó una reducción numérica y se prefijó de antemano que pertenece a la escala ordinal y se establecieron las siguientes siete categorías o niveles de valoración posibles. El ordenamiento mediante números no significa que éstos funcionen plenamente como tales, ya que sólo representan relaciones del tipo "mayor que" o "menor que". No puede saberse si la diferencia entre dos categorías es igual para todos los pares de categorías consecutivas ni tampoco puede sostenerse que el valor " 2 " representa el doble

1 A partir de la distinción realizada por Samaja $(2005,291)$ entre clasificación y diagnóstico, este referido a la ubicación de cierto caso en un clase de una clasificación previamente existente. 
que el "1". Pero sí puede pensarse que las categorías a considerar para el nivel de valoración tienen un orden entre sí y caen en un continuo que muestra la "fuerza" de dicho nivel, desde un "alto grado" hasta el "no se valora". Esto permite el análisis comparativo entre las dimensiones componentes, y en su caso, la comparación entre distintos casos a estudiar.

En principio, se estableció una correspondencia entre las posibles respuestas de las variables y los niveles de valoración prefijados de antemano. De esta manera, cada una de las variables arroja un valor entre alto grado de valoración (2) y no se valora (0). En este estudio, se consideran todas las variables con la misma ponderación.

Para la detectar la relación entre el paradigma cultural y el nivel de receptividad de la cultura de los aspectos emocionales en relación con la creatividad y la innovación (tercer objetivo) se realiza un estudio de caso en profundidad.

Se toman en cuenta las contribuciones metodológicas, que dan lugar al estudio de casos Yin (1994) y Eisenhardt (1989). Al tratarse de un estudio cualitativo exploratorio, y al no estar en juego, en una primera instancia, la cuestión de generalización de los resultados, la cantidad de sujetos queda abierta (Samaja, 2005, 272). Se optó por el diseño de un único caso y de tipo holístico (Yin, 1994).

La elección del caso se justifica por tres características relevantes del mismo. La primera, el nivel de apertura para la participación en la investigación, el que resulta un requisito insoslayable por la profundidad del presente estudio. Por otra parte, este caso forma parte de un estudio de cultura organizacional que incluía un total de diez empresas (Camio, 2012), destacándose éste por su consistencia interna, en términos del nivel de articulación de su paradigma cultural. Esta característica viabiliza un estudio con mayor nivel de especificidad, ya que en esta investigación se consideran las dimensiones culturales relacionadas directamente con la creatividad y la innovación.

En tercer término, se había calculado en esta empresa el Índice de Nivel de Innovación específico para el sector de software (INIs) resultante de etapas previas de investigación (Camio et al., 2013), lo que contribuía positivamente para el análisis de la relación entre la cultura y el nivel de innovación de la empresa en estudio.

Por lo antes expuesto, se entiende que este caso contribuye en buena medida a la generalización del tema en estudio, y a la realización de inferencias lógicas (generalización analítica), lo que permite avanzar en el desarrollo de una teoría que puede ser transferida a otros casos, es decir contribuye a su "transferibilidad" (Maxwell, 1998).

Se trata de una empresa con las características que se describen a continuación; tamaño: PyME; edad de las empresas: con más de cinco años; sector: software y servicios informáticos; ubicación geográfica: Tandil, Provincia de Buenos Aires.

Se construye un protocolo de recogida de datos, con la idea principal de "triangular" o establecer líneas convergentes de evidencia, a partir de la búsqueda de fuentes independientes que apunten a la misma serie de eventos o "hechos" para ampliar la base probatoria.

En el estudio de caso se incluyen distintas fuentes de datos de tipo primario, obtenidos a través de la observación directa, análisis de comunicación institucional (página web, folletería, etc.) y entrevistas en profundidad. Se mantuvieron contactos con todos los miembros de la empresa, y se realizaron entrevistas en profundidad con dos de sus socios fundadores y con uno de los programadores con una antigüedad media en la empresa.

Esta muestra fue escogida a partir de un análisis previo de funciones y niveles jerárquicos en función de las necesidades de información a partir de las definiciones operativas propuestas. Se realizaron preguntas abiertas y se aplicó el cuestionario ad-hoc construido para la descripción general de la cultura, la identificación del grado de articulación del paradigma (GAP), el nivel de asociación con la innovación (NAI) y el nivel de receptividad de la cultura de los aspectos emocionales en relación con la creatividad y la innovación (INCEIC), cuyos componentes se explicitan en la Tabla 1. 
Tabla 1. INCEIC: Variables, sub-variables e indicadores y sus categorías de respuesta ${ }^{2}$

\begin{tabular}{|c|c|c|c|}
\hline $\begin{array}{c}\text { Variable/ } \\
\text { subvariable }\end{array}$ & \multicolumn{2}{|c|}{$\begin{array}{l}\text { Respuestas y su nivel de aso- } \\
\text { ciación con la innovación } \\
(-) \ldots \ldots \ldots \ldots \ldots \ldots \ldots . . . . .(+) \\
\text { barreras impulsores }\end{array}$} & Indicadores \\
\hline \multicolumn{4}{|l|}{ 1. Cultura } \\
\hline $\begin{array}{l}\text { 1.1. Naturaleza humana } \\
\text { (N.H.) }\end{array}$ & Neutra & Buena & $\begin{array}{l}\text { Creencia acerca de si las personas son X o Y (Mc Gregor). } \\
\text { Identificación de héroes o malvados. Justificaciones. Historias relacionadas. } \\
\text { Enunciación de valores y disvalores de la empresa. "Cosas que no se negocian". } \\
\text { Identificaciones de valores impulsores de la innovación. } \\
\text { Identificación de "exitosos" y "fracasados" en la empresa. } \\
\text { Aspectos que se controlan de los empleados. Sistemas implementados. }\end{array}$ \\
\hline $\begin{array}{l}\text { 1.2. Enfoque del tiempo } \\
\text { (E.T.) }\end{array}$ & Pasado & Futuro & $\begin{array}{l}\text { Relato de decisiones recientes e identificación de la importancia concedida al pre- } \\
\text { sente, al pasado o al futuro. } \\
\text { Identificación en el relato "que se cuenta" de la historia de la empresa a "qué tiem- } \\
\text { po" se le da importancia. En dónde se ancla el relato y si se proyecta al futuro. }\end{array}$ \\
\hline $\begin{array}{l}\text { 1.3. Posición frente al } \\
\text { entorno (E.) }\end{array}$ & $\begin{array}{l}\text { Dominante } \\
\text { Dominado }\end{array}$ & En armonía & $\begin{array}{l}\text { Relato de las últimas decisiones y desarrollo de proyectos, ver la articulación con } \\
\text { el medio, si se aplica la idea de innovación abierta, y el desarrollo de redes de } \\
\text { contacto. }\end{array}$ \\
\hline $\begin{array}{l}\text { 1.3. Criterios verificativos } \\
\text { de la realidad (CVR) }\end{array}$ & $\begin{array}{l}\text { Tradición } \\
\text { Procedimiento Legal } \\
\text { Religión/Dogma Moral } \\
\text { Examen científico }\end{array}$ & $\begin{array}{l}\text { Prueba y Error } \\
\text { Conocimiento } \\
\text { Experiencia } \\
\text { Revelaciones } \\
\text { de Autoridades }\end{array}$ & $\begin{array}{l}\text { Identificar los criterios dominantes al momento de resolver conflictos en el proceso } \\
\text { de toma de decisiones estratégicas. } \\
\text { Elección en forma directa de los criterios utilizados para tomar una decisión (de un } \\
\text { listado que contiene la explicación de los mismos). Justificación de la elección. } \\
\text { Relato del proceso que permitía a la gente pensar "que se podía llegar a la decisión". }\end{array}$ \\
\hline $\begin{array}{l}\text { 1.4. Relaciones humanas } \\
\text { (RRHH) }\end{array}$ & $\begin{array}{l}\text { Linealidad } \\
\text { Individualismo } \\
\text { Competencia } \\
\text { Tradición } \\
\text { Bienestar Individual }\end{array}$ & $\begin{array}{l}\text { Colateralidad } \\
\text { Bienestar del grupo } \\
\text { Cooperación Grupal } \\
\text { Jerarquía } \\
\text { Familia }\end{array}$ & $\begin{array}{l}\text { Descripción de incidentes de rebeldía que pudieron suceder en la empresa. Cuál } \\
\text { fue la manera de resolverlos y qué sucedió con los empleados que participaron en } \\
\text { estos incidentes. } \\
\text { Cuestionamiento acerca de lo que se entiende por "conflicto" y si se promueven o } \\
\text { toleran los conflictos en la empresa. } \\
\text { Examinar anécdotas y leyendas de la empresa sobre los "héroes" y los "malvados", } \\
\text { para descubrir la forma en que esas personas se relacionan con otros individuos de } \\
\text { la empresa. } \\
\text { Identificar en el proceso de decisiones, anécdotas y eventos, si estos hacen refe- } \\
\text { rencia a las personas o al grupo, qué resulta más importante y si se desarrollan } \\
\text { relaciones de competencia o de cooperación y en qué grado cada una de estas. }\end{array}$ \\
\hline $\begin{array}{l}\text { 1.5. Relaciones } \\
\text { empresariales } \\
\quad \text { (RREE) }\end{array}$ & $\begin{array}{l}\text { Autocracia } \\
\text { Consultiva }\end{array}$ & $\begin{array}{l}\text { Paternalismo } \\
\text { Participativa }\end{array}$ & $\begin{array}{l}\text { Cuestionamiento acerca de si el número } 1 \text { de la empresa incide fuertemente en la } \\
\text { evaluación de la información. } \\
\text { Identificación de una serie de decisiones importantes adoptadas recientemente. } \\
\text { Identificar, si fueron tomadas por individuos, grupos o ambos, y cómo se ejerció el } \\
\text { poder en el proceso de toma de decisiones. Distinguir el tipo de decisión (estratégi- } \\
\text { ca/operativa) y el nivel de participación. }\end{array}$ \\
\hline \multicolumn{3}{|c|}{$\begin{array}{l}\text { 2. Nivel de receptividad de la cultura } \\
\text { de los aspectos emocionales en relación } \\
\text { con la creatividad (2.1.) y la innovación (2.2) } \\
\text { INCEIC }\end{array}$} & 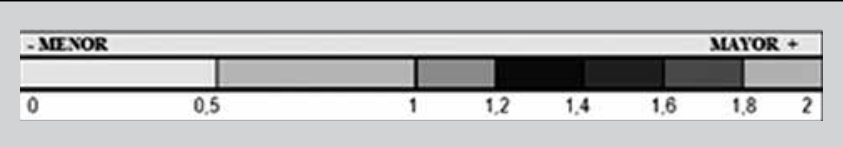 \\
\hline $\begin{array}{l}\text { 2.1.1. Nivel de valoración } \\
\text { del conocimiento } \\
\text { emocional (CEm) }\end{array}$ & No se valora & Grado de Valoración & $\begin{array}{l}\text { Nivel de valoración del conocimiento de los individuos de sus estados de ánimo y } \\
\text { emociones, En este ítem se cuestiona acerca del grado de valoración: } \\
\text { a) No se valora. A lo que se le asigna un valor de } 0 \text { para el indicador. } \\
\text { b) Se valora en baja medida. Lo que equivale a un } 1 . \\
\text { c) Se valora en gran medida. Lo que equivale a un } 2 \text {. }\end{array}$ \\
\hline
\end{tabular}

2 Fuente: Elaboración propia. 


\begin{tabular}{|c|c|c|}
\hline $\begin{array}{l}\text { 2.1.2. Nivel de valoración } \\
\text { de la honestidad emocional } \\
\text { (CEHm) }\end{array}$ & Grado de Valoración & $\begin{array}{l}\text { Nivel de valoración que la gente vaya más allá de escuchar abiertamente y honra- } \\
\text { damente, implica respetar lo que siente intuitivamente y actuar en consecuencia. } \\
\text { a) No se valora. A lo que se le asigna un valor } 0 \text {. } \\
\text { b) Es valorado pero a veces se "gana" más de otra manera, a lo que se le asigna } \\
\text { un valor } 1 \text {. } \\
\text { c) Es valorado como lo más importante en la empresa, a lo que se le asigna un } \\
\text { valor } 2 \text {. }\end{array}$ \\
\hline $\begin{array}{l}\text { 2.1.3. Nivel de valoración } \\
\text { de la retroinformación } \\
\text { emocional (CERm) }\end{array}$ & Grado de Valoración & $\begin{array}{l}\text { Grado en que se valora la capacidad de superar la impulsividad y guiar apropiada- } \\
\text { mente la reacción de las emociones. } \\
\text { a) Se valora que las personas expresen las emociones de manera apropiada superan- } \\
\text { do la impulsividad, a lo que se le asigna un valor } 2 \text {. } \\
\text { b) Es preferible que la gente oculte las emociones o las deje de lado antes de expre- } \\
\text { sarlas frente a las demás personas de la empresa, a lo que se le asigna un valor } 1 \text {. } \\
\text { c) Se acepta que la gente exprese en forma impulsiva sus emociones, a lo que se } \\
\text { le asigna un valor } 0 \text {. }\end{array}$ \\
\hline $\begin{array}{l}\text { 2.1.4. Nivel de valoración } \\
\text { de la empatía en las } \\
\text { relaciones (Emp) }\end{array}$ & Grado de Valoración & $\begin{array}{l}\text { Valoración de la empatía como elemento a desarrollar, la que proviene de la conexión } \\
\text { persona a persona cuando existe un ambiente de confianza. Se propone una grilla } \\
\text { con diferentes afirmaciones vinculadas al concepto de empatía y se pide la elección } \\
\text { en cada caso de una de la respuesta acerca del nivel de valoración: } \\
\text { a) Se valora en gran medida. Se le asigna un valor } 2 \text { a cada sub-ítem y luego se } \\
\text { realiza la suma proporcional con igual ponderación para el ítem. } \\
\text { b) Se valora de mediana medida. Se le asigna un valor } 1 \text { a cada sub-ítem y luego se } \\
\text { realiza la suma proporcional con igual ponderación para el ítem. } \\
\text { c) No se valora. Se le asigna un valor } 0 \text { a cada sub-ítem y luego se realiza la suma } \\
\text { proporcional con igual ponderación para el ítem. }\end{array}$ \\
\hline $\begin{array}{l}\text { 2.2.1. Nivel de desarrollo } \\
\text { del radio de confianza } \\
\text { (AERC) }\end{array}$ & Grado de Valoración & $\begin{array}{l}\text { Se pregunta si se dan las condiciones en la empresa para que la gente confíe en los } \\
\text { que trabajan en la misma. Las respuestas son sí o no y se solicita justificación. A } \\
\text { partir del análisis de las respuestas, éstas se traducen a los siguientes valores a los } \\
\text { fines ilustrativos. } \\
\text { a) Se valora, a lo que se asigna un valor } 201 \text { según el análisis de las justificaciones } \\
\text { expuestas. } \\
\text { b) No se valora, a lo que se le asigna un valor } 0 \text {. }\end{array}$ \\
\hline $\begin{array}{l}\text { 2.2.2. Nivel de valoración } \\
\text { de la intuición en la toma } \\
\text { de decisiones (ALED) }\end{array}$ & $\begin{array}{l}\text { Siempre } \quad \text { Si se puede } \\
\text { Nunca justificar algunos casos }\end{array}$ & $\begin{array}{l}\text { La forma de uso de la intuición en la toma de decisiones en la empresa determina el } \\
\text { aporte al cálculo del INCEIC: } \\
\text { a) Siempre, a lo que se asigna un valor } 0 \text {. } \\
\text { b) En algunos casos, a lo que se asigna un valor } 2 \text {. } \\
\text { c) Se permite lo que se pueda justificar, a lo que se asigna valor } 1 . \\
\text { d) Nunca, a lo que se asigna valor } 0 \text {. }\end{array}$ \\
\hline $\begin{array}{l}\text { 2.2.3. Valoración del } \\
\text { sentido del humor en las } \\
\text { relaciones (ALECHT) }\end{array}$ & Rechazado & $\begin{array}{l}\text { Se releva el nivel de valoración del buen sentido del humor en las relaciones, asig- } \\
\text { nándoles distintos valores: } \\
\text { a) Si es valorado: se le asigna un valor } 2 \text {. } \\
\text { b) Si es aceptado: se le asigna un valor } 1 \text {. } \\
\text { c) Si es rechazado: se le asigna un valor } 0 \text {. } \\
\text { Se solicita la respuesta a la pregunta acerca de cómo se da esta valoración a los fines } \\
\text { de corregir en su caso la apreciación de la valoración en las empresas en estudio. }\end{array}$ \\
\hline $\begin{array}{l}\text { 2.2.4. Trabajo para el logro } \\
\text { de confluencia (ALECTC) }\end{array}$ & $\begin{array}{l}\text { No se trabaja } \quad \text { Sí se trabaja } \\
\text { Solo se realizan esfuerzos aislados }\end{array}$ & $\begin{array}{l}\text { Grado en que se trabaja en la empresa para que las intuiciones, los talentos dispares, } \\
\text { los propósitos y competencias, personas y posibilidades confluyan. Se proponen las } \\
\text { siguientes opciones: } \\
\text { a) Sí se trabaja. Se le asigna un valor } 2 \text {. } \\
\text { b) Sólo se hacen esfuerzos aislados. Se le asigna un valor } 1 \text {. } \\
\text { c) No se trabaja. Se le asigna un valor } 0 \text {. }\end{array}$ \\
\hline
\end{tabular}




\begin{tabular}{|c|c|c|}
\hline $\begin{array}{l}\text { 2.2.5. Tipo de energía } \\
\text { emocional predominante. } \\
\text { (CEEm) }\end{array}$ & $\begin{array}{l}\text { Energía tensa Energía tranquila } \\
\text { Cansancio tenso } \\
\text { Cansancio tranquilo }\end{array}$ & $\begin{array}{l}\text { Forma en que se trabaja en la empresa, y su correspondiente valoración: } \\
\text {-Energía tensa (alta tensión, alta energía). Es habitual que la gente tenga un alto nivel } \\
\text { de energía, aun cuando tenga que enfrentar altos niveles de tensión por muchas } \\
\text { horas de trabajo con un programa de trabajo exigente (se le asigna un valor 0). } \\
\text {-Energía tranquila (baja tensión, alta energía) Es habitual que la gente trabaje sere- } \\
\text { namente, con un ánimo atento, con menor esfuerzo y menor desgaste (se le asigna } \\
\text { un valor 2). } \\
\text {-Cansancio tenso (alta tensión, baja energía) Es común que la gente trabaje en un } \\
\text { estado de cansancio general, con un sentimiento de baja autoestima y de que la vida } \\
\text { es una carga (se le asigna un valor 0). } \\
\text {-Cansancio tranquilo (baja tensión, baja energía) Es común que la gente atraviese } \\
\text { por períodos prolongados en los que tenga que cumplir con una exigente fecha } \\
\text { límite en el trabajo y que luego se abandone y relaje hasta un nuevo desafío (se le } \\
\text { asigna un valor 1). } \\
\text { En el caso de que se opte por más de una alternativa, se considera el valor medio } \\
\text { correspondiente a ambas opciones. }\end{array}$ \\
\hline
\end{tabular}

Dentro de la planificación del trabajo de campo se consideraron los siguientes objetivos:

a. Identificar qué efectos posibles tendrá la intrusión del investigador en el contexto. En relación a este punto se prestó especial atención a propiciar un ámbito de comunicación que permitiera generar confianza y empatía con el entrevistado.

b. Proponer para cada esfera de fenómenos conexos, mecanismos de observación. En algunas dimensiones analizadas, referidas a los aspectos físicos y los aspectos de interrelación observables (Schvarstein, 1997, 168 -200), se puntualizaron los siguientes:

- Área afectiva: Comportamientos indicativos de la intensidad emocional de los vínculos entre las personas. Gritos y susurros, llantos y risas, gestos adustos o distendidos, tensión o relajamiento, miradas que se buscan o que se eluden.

- Comportamiento de los superiores en relación con los subordinados: Cercanía o distancia, cortesía u hostilidad.

- Signos de relación: Modo en que se saludan (se dan la mano, se besan). Se analiza si hay diferencias en función del nivel jerárquico de los sujetos.

- Arquitectura interior: La decoración de los interiores, los colores, el cuidado prestado a la ambientación, los muebles, la iluminación, la temperatura ambiente. Se observa también si hay diferencias arquitectónicas en los sitios destinados a distintos niveles o sectores de la empresa.

- Imagen institucional. Identificación de las marcas, logos, diseños de folletería y páginas institucionales. Qué características tienen, que quieren comunicar.

- Vestimenta: Se analiza si se utiliza o no uniforme, quienes lo usan. Que nivel de formalismo se exhibe en la vestimenta.

- Relaciones de género. Hombres y mujeres. Se observa el trato interpersonal y si surgen diferencias.

- Distribución de los lugares de trabajo: la opacidad o transparencia de los lugares donde se interactúa con los demás, la cantidad y distribución de los muebles, la asignación de maquinarias y equipos, y su relación con el lugar para las personas, quienes tienen ventanas al exterior, y quienes no.

- Existencia de lugares y su relación con el folklore organizacional: Se identifican lugares como la "pecera", espacio más o menos central separado por tabiques vidriados transparentes; la "cueva" es lugar de refugio, y donde nadie ve lo que pasa, la "torre de marfil" donde puede estar encerrada la dirección; la "siberia" es el lugar de destino de los disidentes o marginales, entre otros identificables.

- La relación entre los sujetos y el espacio de trabajo: Identificación de la existencia de elementos que denotan "apropiación" 
del lugar de trabajo, como la foto de la familia en el escritorio, objetos personales exhibidos, etc. La existencia de espacios desnudos, la ausencia de ornamentación, de cuidado, puede revelar una actitud de retirada, una carencia de integración de la persona con la organización.

c. Disponer de mecanismos de registro lo más amplios y fieles posibles de los acontecimientos (ver la influencia en el contexto, su fidelidad y su riqueza).

Se utilizaron los registros escritos en referencia a los cuestionarios elaborados al efecto. Por otra parte se grabaron y desgrabaron las entrevistas en profundidad realizadas.

Una vez finalizada la fase de recogida de datos se realiza el análisis de dicha evidencia vinculándola a las proposiciones planteadas. Se inspecciona, categoriza, tabula y/o recombina dicha información, confrontándola de manera directa con las proposiciones iniciales de la investigación. Los resultados de este proceso se exponen en el punto 6.1. en términos descriptivos y, se ilustran en la Tabla 2 - 3 y en la Gráfica 1.

Para la identificación del grado de articulación del paradigma (GAP), el nivel de asociación con la innovación (NAI) y el Nivel de receptividad de la cultura de los aspectos emocionales en relación con la creatividad y la innovación (INCEIC), las respuestas obtenidas de los distintos entrevistados se computan con la misma ponderación, aunque al momento del análisis de cada una de las variables o sub-variables que componen los índices, se identifican, como un aspecto importante en el análisis, si existen diferencias en las respuestas en función del nivel de responsabilidad y área a la cual pertenecen los entrevistados.

\section{Descripción y análisis de los resultados del caso en estudio}

\subsection{Descripción general del caso en estudio}

La empresa en estudio está dedicada a la provisión de servicios de alta calidad en el área de IT, radicada en la ciudad de Tandil (Argentina). Uno de los princi- pales objetivos de la misma es generar relaciones de mutua confianza y de largo plazo con sus clientes a través de una conducta responsable y transparente.

Se transcriben a continuación la Misión y la Visión, según se publicitan en su página web.

- Misión: "Nuestra determinación es guiar y acompañar a nuestros clientes en su crecimiento, con un fuerte compromiso en la calidad $e$ innovación de las soluciones tecnológicas que brindamos".

- Visión: "Ser proveedores de servicios de IT con presencia en Latinoamérica en los sectores corporativo y gobierno, reconocidos por la calidad de nuestros servicios, basándonos en la innovación continua y la calidad humana de nuestro staff".

La firma ha desarrollado clientes de diferente magnitud, entre los que se encuentran, MoviStar (actualización de la plataforma y benchmark post migración, de base de datos Oracle 9i, sobre sistema operativo Solaris para Movistar Chile), Pami (solución central -Sistema Core- del I.N.S.S.J.P, con arquitectura J2EE, sobre la cual el Instituto basará toda su infraestructura tecnológica central).

Adicionalmente, la empresa ha desarrollado todos los aplicativos con tecnología Mobile del Pami, sobre dispositivos BlackBerry, Aeropuertos Argentina 2000 (participaron en la implementación de Acceso Biométrico a Terminal Retiro y Ezeiza en instalaciones de Aeropuertos Argentina 2000), Terminal de Cargas Argentina (Brindan servicios de Soporte de Infraestructura Oracle -a través de Numen Insight- a la empresa Edcadassa -TCA-, Terminal de Cargas Argentina), empresa que se dedica, entre otras cosas al manejo de cargas de importación y exportación, servicios de Courier internacional, etc. Desde su NOC (Network Operations Center) la empresa brinda servicios de Monitoreo de todas las bases que Terminal de Cargas Argentina tiene distribuidas en el mundo.

La empresa funciona también como Software Factory, dedicada a la producción de aplicaciones y componentes de software. Se especializa en proyectos de modernización de aplicaciones desarrolladas 
en Oracle Forms y Reports en cualquiera de sus versiones. Por otra parte, brinda Soluciones tecnológicas a sus clientes, presentándose como socio estratégico en sus negocios, ocupándose de las tareas que no son parte de sus core-business.

La empresa ofrece modalidades de contratación a la medida de sus clientes, que van desde el outsourcing hasta el outtasking de su área de IT, con una gran variedad de servicios entre los que se encuentran: Delivery Services, Soporte de Infraestructura. Desarrollan estos proyectos tanto en el país como en el extranjero, principalmente en los sectores de Salud, Gobierno y AgroNegocios.

Sus servicios incluyen: Dirección y Liderazgo de Proyectos, Asesoramiento en Tecnología y Procesos, Consultoría en StartUp de proyectos, Entrenamiento y Capacitación, Asistencia Técnica en Bases de Datos, Herramientas y entornos de desarrollo, Hardware, Networking, Storage, Middleware, Software para Integración de Aplicaciones, entre otras.

El grupo de trabajo está formado por ocho personas, de las cuales tres son socios. Cuatro de ellos tienen formación de ingenieros en sistemas, y el resto estudiantes avanzados de la carrera. El gerente tiene cursado un MBA. Es una empresa joven que se constituyó como Sociedad de Responsabilidad Limitada en el 2008, aunque informalmente los socios han trabajado juntos en empresas/proyectos anteriores.

La conducción de la empresa la realizan los tres socios (lo denominan "Triunvirato") distinguiéndose en su nivel siguiente de estructura tres áreas de actividades, Responsables de servicios (del cual depende un profesional), Responsable de Producción (con cuatro personas profesionales/estudiantes avanzados a cargo) y el área de Comercialización y Administración (encargada de hacer contactos con los clientes, aspectos de administración y demás aspectos de comercialización a cargo en gran medida de los socios).
Se eligió el nombre que denota que los individuos buscan formar un grupo "que cohesione bien", hacer un trabajo inteligente donde cada uno sabe lo que tiene que hacer.

Entre las últimas decisiones que se tomaron se decidió "apuntar" a empresas multinacionales y gubernamentales con productos más específicos teniendo en cuenta la necesidad en el mercado que marca Oracle.

Es de destacar que de la aplicación del INIs (Indice de Nivel de Innovación específico para empresas de software ${ }^{2}$ ) surge que la empresa presenta un nivel de Innovación Medio/Alto, con los siguientes resultados en sus variables componentes: Capacidades para la innovación Medio/Alto, Resultados de la innovación Alto/Muy Alto e Impactos Bajo/Medio.

\subsection{Aplicación de la metodología de diagnóstico al caso en estudio}

En el estudio se expone en primer término, el grado de articulación del paradigma cultural, en caso de poderse identificar (GAP), utilizándose las categorías: no articulado (no se identifica paradigma), parcialmente articulado, articulado, fuertemente articulado.

La empresa en estudio, aunque muy joven, ha conformado una cultura sólida y consistente. Una de las causas puede atribuirse a la experiencia conjunta de los socios en trabajos anteriores. Además, estos tienen importantes puntos de contacto en sus visiones personales. La cultura de la empresa se reafirma en forma consciente y sistemática en el proceso de selección de los nuevos integrantes de la empresa, la que se "pondrá a prueba" con el devenir de su historia.

Una presunción que se puede considerar central es la referida a cómo se entiende a la naturaleza del

2 El INIs resulta de varios años de investigación en el marco del proyecto de "Innovación y Modalidades de Gestión" del Centro de estudios en administración (CEA) de la Universidad del Centro de la Provincia de Buenos Aires. Se exponen en trabajos anteriores, el modelo y la operacionalización en el citado indicador (Camio et al., 2013). Se consideran 9 categorías de respuesta. 
género humano (buena, mala o neutra). Existe un acuerdo entre todos los encuestados acerca de que en la empresa se entiende a las personas como buenas por naturaleza. No se realizan controles formales como de horarios, ya que se trabaja por tareas y de manera flexible. Se considera la posibilidad del teletrabajo como opción.

Los valores o las "cosas que no se negocian en la empresa" que surgen de las entrevistas son:

- El profesionalismo: relacionado con la capacitación de los miembros, que sean confiables. Se valora el "esfuerzo puesto en las cosas", y la cohesión del grupo por encima de la excelencia del profesional individual.

- La "buena onda", "tratarse bien", ser respetuosos entre ellos, el compañerismo, la calidad humana.

- Entender que los productos entregados son una solución innovadora para el cliente, la responsabilidad (compromiso de entrega con calidad), quieren que los clientes los vean como una empresa sólida, muy bien formada.

Se identifican aspectos que reafirman los valores antes descriptos a través de la observación del lugar, de la "manera en cómo trabaja", y de la comunicación que realizan (página web):

- Exhiben los certificados de los cursos realizados por distintos integrantes de la empresa, no sólo los de los socios.

- Se respira un muy buen clima de trabajo, toman mate, se comunican en forma distendida. - Se evidencia prolijidad y cuidado en el uso de los espacios. Consideran algunos elementos del feng shui en la decoración y ambientación del lugar. Algunos de ellos, socios o empleados, usan una chomba informal con el logo de la empresa. Para fin de año realizaron una jornada en una quinta que alquiló la empresa en donde compartieron el día todos los integrantes de la empresa.

- La página web muestra todos sus productos y clientes, comunicando los valores que ellos señalan en este punto.
En relación a la atribución del éxito o el fracaso, no se realizan muchas distinciones en este sentido. Si se identifican personas exitosas, se refieren al conocimiento que pudieron adquirir (lo que los constituye en referentes para determinado proyecto por la tecnología que este involucra). En relación a esto las historias que se cuentan, se vinculan al nacimiento de la empresa y a la forma en que la "armaron", trabajando todos como obreros para acondicionar el edificio.

La forma adoptada para el proceso de toma de decisiones revela qué áreas de dominio de la realidad resultan más importantes y cómo se determina esta. En tal sentido, las decisiones estratégicas son tomadas por los socios, consultando a los otros integrantes. Si surge alguna dificultad o tecnología que desconocen, los empleados capacitados y los socios deciden cuál es la mejor manera de resolver la situación. Surge con cierta claridad que las decisiones no se toman a partir de la tradición, ni por dogma alguno. Resultan determinantes las opiniones de los socios.

Por otra parte y en relación con la generación de ideas creativas o innovadoras, se escuchan y analizan las que surgen del personal y se le otorga la autoridad necesaria para llevarlas adelante. El sistema de reconocimiento al personal que innova no se encuentra muy desarrollado, hay un reconocimiento de tipo verbal y grupal en reuniones en las que participan todos los integrantes de la empresa.

La empresa utiliza procedimientos propios de la tecnología de sistemas, aunque consideran que se requiere implementar mejora en las metodologías de trabajo.

El término "conflicto" se atribuye a un "problema, una discusión". En algunos casos en los que existen puntos de vista diferentes, éstos se consideran positivos. No surgen de ellos peleas o cuestiones de esta índole. No se promueven, sí se toleran, los conflictos en la empresa.

A partir del planteo de las decisiones importantes se identifica, con relación a la naturaleza del tiempo, la orientación hacia el futuro, reflejada en la frase "nosotros siempre miramos para el futuro". Se piensa 
siempre en proyectos, que se desarrollan en simultáneo por distintos grupos de personas. El flujo de trabajo fluye sin rutina fija, y hay cierta autonomía para el manejo del tiempo de cada uno de los integrantes de la empresa.

Con respecto a la orientación de la naturaleza de la realidad y la verdad, se utiliza el diálogo y el consenso para la toma de decisiones. Es muy importante el "saber" técnico. Éste se valora por sobre la autoridad formal, sin desconocer el rol de los socios en las definiciones estratégicas.

Las relaciones humanas se entienden como de cooperación, haciendo fuerte hincapié en la cohesión del grupo y en la armonía en las relaciones entre todos los integrantes de la empresa.

Con respecto a la naturaleza de las relaciones empresariales, las decisiones de tipo estratégico son responsabilidad de los socios, y las decisiones operativas se toman de forma participativa.

A continuación, se analiza el nivel de asociación de las respuestas obtenidas en relación a la capacidad para innovar de la firma. Se exponen en la Tabla 2 las respuestas a las presunciones y su nivel de vinculación, identificando aquellas que actúan como impulsoras $(+)$ y aquellas que actúan como barreras (-) para la innovación (en relación a la clasificación expuesta en la Tabla 1). Se exponen los resultados acerca de la manera en que se entiende la naturaleza humana (N.H.), cuál es el enfoque del tiempo (E.T.), la posición frente al entorno, los criterios verificativos de la realidad y verdad (C.V.R.), cómo se entienden las relaciones humanas $(\mathrm{RRHH})$ y las relaciones empresariales (RREE).
Tabla 2. Descripción y exposición de los resultados del análisis del paradigma cultural ${ }^{3}$

\begin{tabular}{|c|c|c|c|c|c|}
\hline \multicolumn{6}{|c|}{$\begin{array}{l}\text { I: CULTURA } \\
\text { A) GAP: Fuertemente articulado } \\
\text { B) NAI: MUY ALTO }\end{array}$} \\
\hline NH & ET & PE & CVR & RRHH & RREE \\
\hline Buena & Futuro & En armonía & $\begin{array}{l}\text { Conoci- } \\
\text { miento } \\
\text { Autoridad }\end{array}$ & $\begin{array}{l}\text { Coopera- } \\
\text { ción } \\
\text { Grupal }\end{array}$ & $\begin{array}{l}\text { Estratégicas: } \\
\text { Consultiva } \\
\text { Técnicas: } \\
\text { Participativas }\end{array}$ \\
\hline \multicolumn{6}{|c|}{$\begin{array}{l}\text { REFERENCIAS: } \\
\text { GAP: Grado de articulación del paradigmas. } \\
\text { NAI: Nivel de asociación con la innovación. } \\
\text { NH: Naturaleza Humana } \\
\text { ET: Enfoque del tiempo } \\
\text { PE: Posición frente al entorno } \\
\text { CVR: Criterios verificativos de la realidad } \\
\text { RRHH: Relaciones Humanas } \\
\text { RREE: Relaciones empresariales }\end{array}$} \\
\hline
\end{tabular}

A continuación, se exponen los resultados del cálculo de INCEIC, el que surge de la ponderación en igual medida de las sub-variables que lo componen, las que se relacionan de manera más directa con la creatividad, a saber: el nivel de valoración del conocimiento emocional (CEm), el nivel de valoración de la honestidad emocional (CEHm), el nivel de valoración de la retroinformación emocional (CERm) y el nivel de valoración de la empatía en las relaciones (Emp).

Por otra parte, se exponen las variables directamente relacionadas con la innovación: el nivel de desarrollo del radio de confianza (AERC), el nivel de valoración de la intuición en la toma de decisiones (ALED), la valoración del sentido del humor en las relaciones (ALECTH), el trabajo para el logro de confluencia (ALECTC) y el tipo de energía emocional predominante en la empresa (CEEM).

3 Fuente: Elaboración propia.

4 Fuente: Elaboración propia. 
Tabla 3. Descripción y exposición de los resultados del INCEIC $^{4}$

\begin{tabular}{|c|c|c|c|c|}
\hline INCEIC & CEm & CEHm & CERm & Emp \\
\hline ALTO & ALTO & ALTO & MUY ALTO & ALTO \\
\hline AERC & ALED & ALECTH & ALECTC & CEEM \\
\hline $\begin{array}{l}\text { MUY } \\
\text { ALTO }\end{array}$ & MEDIO & MUY ALTO & $\begin{array}{l}\text { MUY } \\
\text { ALTO }\end{array}$ & MEDIO ALTO \\
\hline \multicolumn{5}{|c|}{$\begin{array}{l}\text { REFERENCIAS: } \\
\text { INCEIC: Nivel de receptividad de la cultura de los aspectos } \\
\text { emocionales en relación con la creatividad (2.1.) y la innovación (2.2) } \\
\text { CEm = Nivel de valoración del conocimiento emocional } \\
\text { CEHm = Nivel de valoración de la honestidad emocional } \\
\text { CERm = Nivel de valoración de la retroinformación emocional } \\
\text { Emp = Nivel de valoración de la empatía en las relaciones } \\
\text { AERC = Nivel de desarrollo del radio de confianza } \\
\text { ALED = Nivel de valoración de la intuición en la toma de decisiones } \\
\text { ALECHT = Valoración del sentido del humor en las relaciones } \\
\text { ALECTC = Trabajo para el logro de confluencia } \\
\text { CEEm = Tipo de energía emocional predominante. }\end{array}$} \\
\hline
\end{tabular}

Del análisis de los resultados obtenidos, se observan valores similares correspondientes a las respuestas de todos los entrevistados. No se identifican diferencias importantes entre las respuestas de socio-gerente y el resto de los entrevistados, aunque se destacan valores superiores del primero con respecto al resto.

En la variable nivel de valoración del conocimiento emocional (CEm) se obtiene un valor alto, en el nivel de valoración de la honestidad emocional (CEHm) el valor resulta con un grado alto al igual que en el caso de la retroinformación emocional (CERm) y el nivel de valoración de la empatía en las relaciones (Emp).

En la evaluación del nivel de desarrollo de radio de confianza (AERC) resultan respuestas con valores máximos en todos los entrevistados. Esto resulta importante, ya que cuando confiamos lo suficiente para dar pasos al frente nos inspiramos e inspiramos a los demás. Se considera a la confianza como una aptitud emocional, algo que debemos sentir y actuar de acuerdo a ello. Las personas desarrollan su radio de confianza cuando tienen facilidad para hablar con extraños y acoger diferencias y desacuerdos de una manera abierta, no rígida, viendo en ellos fuentes de posibles conexiones e ideas que puedan resultar constructivas y valiosas.

Para la evaluación del grado de aceptación del uso de la intuición en la gestión de las empresas estudiadas, se señala que ante ciertas situaciones (excepcionales y no vinculadas con la necesidad de la rutina) es necesario dejar que la intuición guíe el comportamiento. En este aspecto se obtiene una valoración media en el caso en estudio.

El cuestionamiento permanente de los supuestos que otros aceptan requiere ser liderado, para que los individuos tengan el valor de asumir el riesgo creativo. Desde la dimensión emocional requiere el desarrollo de distintos aspectos que permitan el crecimiento tanto individual como la reunión de las capacidades y talentos de todos los participantes en la empresa. En este punto se valora el sentido del humor en las relaciones (ALECTH), el grado en que se trabaja en la empresa para el logro de la confluencia para la transformación creativa (ALECTC) y el tipo de energía emocional que predomina en la empresa (CEEm).

Se señala al humor como la conducta más significativa de la inteligencia humana, la que aumenta la energía y fomenta el flujo intuitivo ya que la alegría es irreverencia y hace al cambio menos atemorizante. Se obtiene la mayor valoración en el ítem ALECTH.

Ante la pregunta de si se trabaja en la empresa para que las intuiciones, los talentos dispares, los propósitos y competencias, personas y posibilidades confluyan, en todos las respuestas se registró que sí se trabaja para esto, por lo que este indicador (ALECTC) arroja el máximo valor.

El tipo de energía emocional (CEEm) que predomina resulta vital para la generación de flujo intuitivo que contribuye en el decidir. No obstante no haber acuerdo entre todos los entrevistados, la mayoría de los entrevistados consideran que en la empresa se trabaja con Energía tranquila (baja tensión, alta energía) ya que es habitual que la gente trabaje serenamente, con un ánimo atento, con menor esfuerzo y menor desgaste.

4 Fuente: Elaboración propia. 
Gráfica 1. Resultados INCEIC y componentes ${ }^{5}$

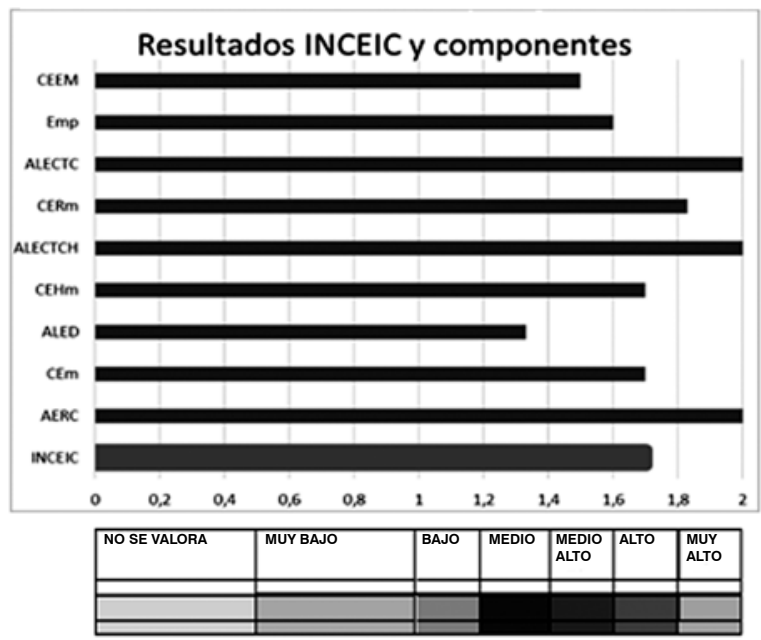

\section{Consideraciones finales}

A partir de la estrategia exploratoria aplicada se puede dar respuesta a los objetivos planteados para este trabajo. A continuación se exponen de manera sintética algunas observaciones respecto de cada uno de los objetivos planteados anteriormente:

Se definieron los lineamientos para la identificación de paradigmas culturales a partir de las presunciones constituyentes y su interrelación, teniendo en cuenta su contribución a la generación de la innovación. Se identificó un paradigma fuertemente articulado (GAP) y un nivel muy alto de asociación con la innovación (NAI). A partir de la discusión teórica anteriormente planteada se pudo identificar en la empresa estudiada un alto nivel de coherencia entre las respuestas a las presunciones culturales planteadas por Schein y cuya operacionalización se aplica en el desarrollo del presente trabajo.

Lo anterior guarda relación con la respuesta a la presunción de la naturaleza humana como buena, afirmado por los valores claves y los artefactos culturales identificados. Contribuyen además el diálogo y el consenso como forma de dar sentido a la verdad y la realidad, y la valoración del saber técnico; además de la defensa de la cohesión del grupo y, la consulta y la participación en la toma de decisiones. El enfoque del tiempo en el futuro potencia las decisiones para la innovación.

Por otra parte, la búsqueda de armonía en relación con el contexto plantea una postura que viabiliza la concreción de diferentes tipos de intermediación requeridos, como son la construcción de redes, el sostenimiento del aprendizaje social y la gestión de la dinámica de poder y los conflictos (Leeuwis \& Aarts, 2011).

Se aplicó el índice para evaluar el nivel de receptividad de la cultura de los aspectos emocionales para la innovación (INCEIC) y a partir de las entrevistas realizadas se obtuvo un alto valor en este índice general, y valores muy altos para el nivel de retroinformación emocional, el nivel de desarrollo del radio de confianza, el nivel de valoración del sentido del humor en las relaciones y el trabajo para el logro de la confluencia. Resulta alto el nivel de valoración de la intuición en la toma de decisiones y medio-alto el tipo de energía emocional predominante.

A partir de la metodología propuesta se pudo detectar la relación entre el paradigma cultural con muy alto nivel de asociación con la innovación y un nivel alto de receptividad de la cultura de los aspectos emocionales para la innovación (INCEI). Lo anterior guarda relación con lo receptado de otros estudios (Choi et al., 2010), ya que en el caso en estudio los miembros de la empresa comparten las evaluaciones tanto cognitivas como emocionales acerca de la innovación.

Por otra parte, se identifica un fuerte apego a la identidad y las características salientes de la organización, por la cual los participantes están juntos por beneficios mutuos y vínculos emocionales (Huy, 1999), lo que contribuye en la empresa al desarrollo de capacidad emocional y contribuye al desarrollo de sus capacidades de aprendizaje e indirectamente a su capacidad de innovar en productos y a su performance (Akgün et al., 2007).

5 Fuente: Elaboración propia. 
La aplicación en el caso en estudio del cálculo del INIs con valores Medio/Alto y los resultados en el INCIEC apoyan la evidencia empírica de que las variables a nivel individual como la creatividad y las emociones positivas en último instancia, influyen en los resultados a nivel organizacional como la innovación (Baron \& Tang, 2011).

Para concluir, se entiende que con la metodología propuesta se amplía el enfoque de análisis de la cultura organizacional ya que se incorpora a un análisis racional la dimensión emocional; además, se entiende, profundiza e enriquece el análisis porque permite el enfoque en profundidad en cada una de las dimensiones.

La replicación de esta metodología en otros casos de estudio con características similares y/o distintivas, contribuirá a la generación de nuevas evidencias, lo que motiva nuevos estudios a emprender. Se espera que esta propuesta resulte una herramienta que enriquezca el abordaje tanto docente como profesional. La operacionalización propuesta permitiría su aplicabilidad, pero un objetivo aunque de tipo inicial resultaría más importante aún, el destacar los aspectos emocionales en la agenda de temas para la gestión de una cultura de la innovación.

\section{Referencias}

Akgün, A., Keskin, H., Byrne, J. \& Aren, S. (2007). Emotional and learning capability and their impact on product innovativeness and firm performance. En: Technovation, 27: 501-513.

Ashforth, B. \& Humphrey, R. (1995). Emotion in the workplace: a reappraisal. En: Human Relations, 48: 97-125.

Averill, J. (1999). Individual Differences in Emotional Creativity: Structure and Correlates. En: Journal of Personality, 67(2): 331-371.

Baranger, D. (1995). La matriz de datos. Construcción y análisis de datos. Posadas, Argentina: Editorial Universitaria de Misiones.

Baron, R. \& Tang, J. (2011). The role of entrepreneurs in firmlevel innovation: Joint effect of positive affect, creativity, and environmental dynamism. En: Journal of Business Venturing, 26: $49-60$

Brief, A. \& Weiss, H. (2002). Organizational Behavior: Affect in the Workplace. En: Annual Reviews of Psychology, 53: 279-307.

Camio M. (2012). Cultura Organizacional: Emociones y sentido de vida. Saarbrücken, Alemania: Editorial Académica Española de AV Akademikerverlag GmbH \& Co. KG.

Camio, M., Romero, M. \& Álvarez, M. (2013). Medición del Nivel de Innovación en Empresas del Sector de Software. ALTEC 2013 - XV Congreso Latino-Iberoamericano de Gestión de Tecnología. Porto, Octubre.
Cartwright, S. \& Pappas, C. (2007). Emotional intelligence, its measurement and implications for the workplace. En: International Journal of Management Reviews, 9 (4).

Conger, J. (1989). The charismatic leader. San Francisco: Jossey-Bass.

Cooper, R. \& Sawaf A. (1998). La Inteligencia Emocional aplicada al Liderazgo y las Organizaciones. Bogotá: Editorial Norma.

Coté, S. (2005). A social interaction model of the effects of emotion regulation on work strain. En: Academy of Management Review, 5 (3): 509-530.

Cotec - Fundación para la innovación tecnológica (2006). Marco de referencia de innovación. Madrid: Ed. Club de excelencia en gestión.

Csikszentmihalyi, M. (2011). Creatividad: El fluir y la psicología del descubrimiento y la invención. Barcelona: Ed. Paidós Transiciones.

Chesbrough, H. (2003). Open Innovation: The New Imperative for Creating and Profiting from Technology, Boston, MA: Harvard Business School Press.

Choi, J., Sung, S., Lee, K. \& Cho, D. (2010). Balancing cognition and emotion: Innovation implementation as a function of cognitive appraisal and emotional reactions toward innovation. En: Journal of Organizational Behavior, 32: 107-124.

Damasio, A. (2001). El error de Descartes. Barcelona: Editorial Crítica.

Damasio, A. (2000). Sentir lo que sucede: cuerpo y emoción en la fábrica de la conciencia. Santiago de Chile: Editorial Andrés Bello.

Dávila, A. \& Martínez, N. (1999). Cultura en organizaciones latinas. México DF: ITESM, Siglo Veintiuno Editores.

Elster, J. (2001). Sobre las pasiones: emoción, adicción y conducta humana. Barcelona: Ediciones Paidós S.A.

Eisenhardt, K. (1989). Building Theories from Case Study Research. En: Academy of Management Review, 14(4): 532-550.

Fisher, C. \& Ashkanasy, N. (2000). The emerging role of emotions in work life: an introduction. En: Journal of Organizational Behavior, 21 (2): 123-129.

Fitness, J. (2000). Anger in the workplace: an emotion script approach to anger episodes between workers and their superiors, co-workers and subordinates. En: Journal of Organizational Behavior, 21 (2): 147-162.

Helfat, E., Finkelstein, S., Mitchell, W., Peteraf, M., Singh, H., Teece, D. \& Winter, S. (2007). Dynamic capabilities: Understanding strategic change in organizations. Malden, MA: Blackwell Publishing.

Hirvikoski, T. \& Diz, H. (2009). Developing a conceptual framework for the human side of innovation with particular reference to senior citizens in innovation ecosystems. Disponible en http:// sendai-cyber.icr-eq.co.jp/cluster/program/program02/ files/ pgm02_02_01.pdf

Huy, Q. (1999). Emotional capability, emotional intelligence, and radical change. En: Academy of Management Review, 24(2): 325-345.

Jerez, P., Céspedes, J. \& Valle, R. (2005). Organizational learning capability: a proposal of measurement. En: Journal of Business Research, 58 (6): 715-725.

Kahneman, D. (2011). Thinking, fast and slow. New York: Farrar, Straus and Giroux.

Kantis H. (2004). Desarrollo emprendedor: América Latina y la experiencia internacional. Banco Interamericano de Desarrollo Bogotá: Editorial Nomos S.A.

Lang, J. \& Lee, C. (2010). Workplace humor and organizational creativity. En: The International Journal of Human Resource Management, 21 (1): 46-60. 
Leeuwis, C. \& Aarts, N. (2011). Rethinking communication in innovation processes: creating space for change in complex systems. En: The Journal of Agricultural Education and Extension, 17 (1): 1-13.

Lessem, R. (1992). Gestión de la Cultura Corporativa. Madrid: Ediciones Díaz de Santos, S.A.

Lim, H. \& Choi, J. (2009). Testing an alternative relationship between individual and contextual predictors of creative performance. En: Social Behavior and Personality: an international journal, 37(1): 117-136

Lundvall, B. \& Maskell, P. (2000). Nation states and economic development: from national systems of production to national systems of knowledge creation and learning. En Clark, G., Nahapiet, J. \& Gertler, M. (Eds). The Oxford handbook of economic geography. Oxford: Oxford University Press: 353-372.

Maxwell, J. A. (1998). "Designing a qualitative study". En: Bickman, L. \& Rog, D. J. (eds.). Handbook of Applied Social Research Methods, Sage Publications, Thousand Oaks: 69-100.

Morcillo, P. (1997). Dirección estratégica de la tecnología e innovación. Madrid: Editorial Civitas.
Nayak, R. \& Ketteringham, J. (1986). Break- throughs! Mercury Books, London.

Rodríguez, D. (2006). Gestión organizacional. México DF: Alfaomega. Samaja, J. (2005). Epistemología y Metodología. Buenos Aires, Argentina: Eudeba.

Sandberg, B. (2007). Enthusiasm in the Development of Radical Innovations. En: Creativity and Innovation Management, 16 (3).

Schein, E. (1988). La cultura empresarial y el liderazgo. Barcelona: Editorial Plaza y Janes.

Schein, E. (2004). Organizational Culture and Leadershi. San Francisco: Jossey - Bass Business \& Management.

Schvarstein, L. (1997). Psicología social de las organizaciones. Buenos Aires: Editorial Paidós.

Yin, R. (1994). Case study research. Design and methods. London: SAGE.

Yoguel, G. \& Boscherini, F. (1996). La capacidad innovativa y el fortalecimiento de la competitividad de las firmas: El caso de las Pymes exportadoras argentinas. CEPAL, Documento de Trabajo $\mathrm{N}^{\circ} 171$. 